\title{
Moisture Recovery - A Dynamic Modelling Approach
}

\author{
Martin Kremer $^{1, *}$, Paul Mathis ${ }^{1}$, and Dirk Müller ${ }^{1}$ \\ ${ }^{1}$ RWTH Aachen University, E.ON Energy Research Center, Institute for Energy Efficient Buildings and Indoor Climate, Germany
}

\begin{abstract}
With rising insulation standards, the use of mechanical ventilation, especially in non-residential buildings, is becoming increasingly relevant. To ensure thermal comfort and avoid health problems for people in the room, cost- and energy-intensive humidification of the supply air is necessary. The use of moisture recovery systems can thus significantly reduce the energy consumption of ventilation systems. Despite this energy-saving potential, moisture recovery systems are rarely used in ventilation systems. To forecast the efficiency of moisture recovery systems in partial load operation and under different climatic conditions, a dynamic model of a membrane-based enthalpy exchanger was developed in the object-oriented modelling language Modelica. The model is based on the solution diffusion model, a quite common approach. In contrast to the models found in the literature, the sorption process is not assumed to be in equilibrium state. Rather, as a first approach the membrane's permeance, consisting of the solubility and diffusion coefficient, is modelled with a linear dependency on the moisture difference between the two incoming airflows.

A parameter fitting has been carried out with experimental data to determine the unknown material parameters. The model containing the fitted parameter set was validated using different experimental data.
\end{abstract}

\section{Introduction}

Maintaining the thermal comfort in non-residential buildings is the task of Heating, Ventilation and Air Conditioning (HVAC) systems. Next to heating and cooling processes the air humidification and dehumidification is necessary to provide comfortable air conditions in a certain building. These processes are energy intensive. For humidification, water has to be transformed into gaseous state and for dehumidification, the air is usually cooled below the dew point and then reheated.

Moisture recovery systems can provide a comfortable indoor air humidity while reducing the energy consumption for air conditioning. One common moisture recovery system is the membrane-based enthalpy exchanger.

Several researchers have developed models to predict the efficiency of membrane-based enthalpy exchangers (cf. e.g. [1, 2]). These researchers use the sorption isotherms to describe the sorption process at the surface of the membrane. This is only valid for steady-state exchange processes and therefore does not fit for a dynamic modelling approach. Koester [3] uses the solution diffusion model to describe the moisture transport through a dense membrane. On contrary to other researches, the author does not model the sorption process directly, but uses an overall membrane permeance. As this permeance is as constant, the model does not provide any dependence on the moisture difference between exhaust and supply air.

\footnotetext{
Corresponding author: mkremer@eonerc.rwth-aachen.de
}

In this paper, a dynamic model of a membrane-based enthalpy exchanger is developed using the solution diffusion model and a membrane's permeance with a linear dependency on the moisture difference between the two incoming airflows.

\section{Membrane-based Enthalpy Exchanger}

The experimental investigations for this paper were executed using a membrane-based enthalpy exchanger of the company Zehnder (see Fig. 1), which is used in decentralised ventilation units.

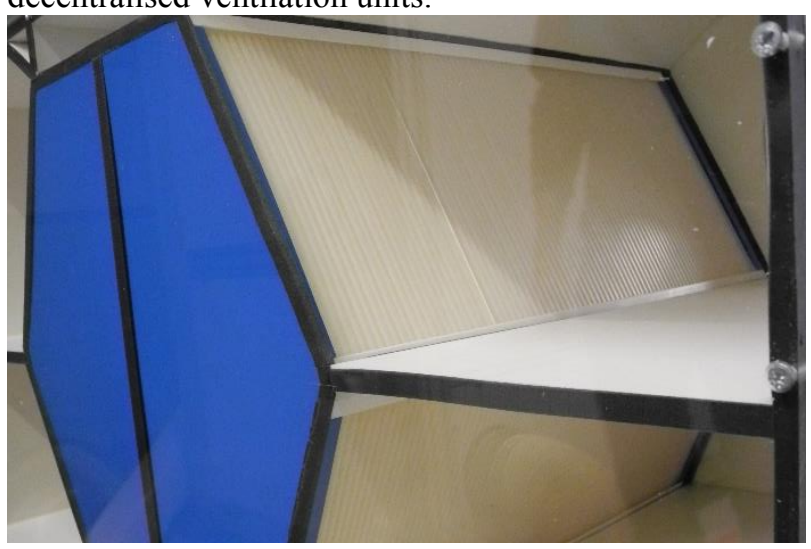

Fig. 1. Membrane-based enthalpy exchanger

The enthalpy exchanger was installed in a test box (see Fig. 1) to separate the two airsides from each other. The air inlets into the box were round. As a result, the flow expands from a diameter of $150 \mathrm{~mm}$ to a rectangular 
cross-section of $450 \times 196 \mathrm{~mm}$. We installed a perforated plate to homogenise the flow at the two inlets. Temperature and relative humidity were measured at the inlets inside the test box. The relative humidity and the related temperature at the outlets were measured further downstream outside the test box. As the absolute humidity did not change along the path from outlet to measurement point, it is valid to calculate the absolute humidity at the outlets from the measured relative humidity and temperature further downstream. In addition to that, the outlets' temperature was measured directly behind the enthalpy exchanger at five different positions of the crosssection, which is pointed out in Fig. 2 . The temperatures were compared in order to monitor the homogeneity of the flow. The outlet temperature was calculated as mean value of the five measured temperatures.

\section{Sensor positions}

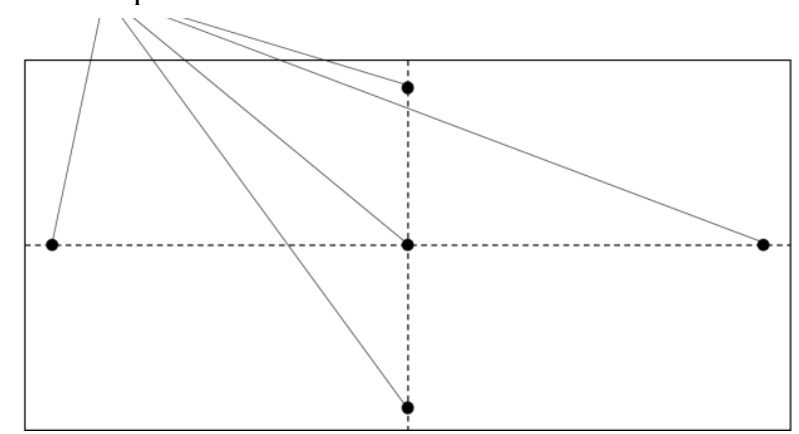

Fig. 2 Sensor positions for temperature measurement at the enthalpy exchanger's outlets

We examined the enthalpy exchanger for different temperature and humidity differences between the two incoming airflows. In addition to that, we investigated the influence of different volume flow rates through the enthalpy exchanger. The test specimen was supplied via a precision air-conditioning unit, which controls both temperature and humidity with high stability and accuracy. Two different conditioning lines are used to set different air conditions for the feed and permeate flow.

\section{Modelling}

Modelling and simulation is done using the objectorientated language Modelica in combination with the software Dymola $[4,5]$. We used the media model of moist air implemented in the AixLib, a Modelica library developed at the Institute for Energy Efficient Buildings and Indoor Climate [6].

The model consists of three sub-models. The membrane and the two air ducts are described in submodels and connected by fluid and heat ports implemented in the Modelica library [5]. The sub-models are discretized in flow direction. Thus, it is possible to map the flow conditions such as co-current or countercurrent flow directly by connecting the air ducts' ports in the same direction or in the opposite direction to the membrane's ports.

\subsection{Modelling of heat and moisture transfer}

The heat and moisture transfer process consists of five sub-processes. In both airflows, convective heat and moisture transfer processes take place. They can be modelled using the analogy between heat and mass transfer, as well as a Nusselt correlation. The water vapour is absorbed at the membrane's surface and the sorption heat is released. The heat is conducted through the membrane and the water vapour diffuses through the membrane. On the opposite surface of the membrane, the water vapour is desorbed requiring the sorption heat.

The solution diffusion model summarizes the sorption and diffusion processes to one process described by the permeability $P_{i}$, which is a material parameter and consists of the diffusion coefficient and the sorption coefficient [7]. This reduces the complex process of sorption, diffusion and desorption to a simple transport equation to describe the moisture transfer through the membrane (eq. (1)).

$$
\dot{m}_{\mathrm{Steam}, i}=A_{\mathrm{Mem}, i} \cdot P_{i} \cdot \frac{\left(p_{i, f}-p_{i, p}\right)}{\delta_{\mathrm{Mem}}}
$$

The water vapour's mass flow rate $\dot{m}_{\text {Steam }, i}$ driven by the difference between the partial pressure of water vapour in the feed $p_{i, f}$ and the permeate stream $p_{i, p}$. Next to the driving potential, the membrane's geometry in form of the surface area $A_{\mathrm{Mem}, \mathrm{i}}$ and the thickness $\delta_{\mathrm{Mem}}$ influences the mass flow rate. The membrane's permeability is declared as an input connector of the membrane sub-model. In this way, it is possible to declare a moisture dependent permeability later on. The transfer model is discretized in flow direction.

For the heat transfer $\dot{Q}_{i}$ through the membrane, the common equation for heat conduction is used (see eq. (2)). The membrane's thermal conductivity $\lambda_{\text {Mem }}$ is defined in a first approach as a constant parameter. The driving potential for the heat conduction is the difference between the feed temperature $T_{i, f, m}$ and the permeate temperature $T_{i, p, m}$ at the membrane's surfaces.

$$
\dot{Q}_{i}=A_{\mathrm{Mem}, i} \cdot \lambda_{\mathrm{Mem}} \cdot \frac{\left(T_{i, f, m}-T_{i, p, m}\right)}{\delta_{\mathrm{Mem}}}
$$

The convective heat transfer for laminar flow in rectangular ducts can be described by the Nusselt correlation developed by Muzychka and Yovanovich [8].

The convective heat transfer coefficient $\alpha$ is calculated from the Nusselt number $N u$ using the thermal conductivity of air $\lambda_{\text {air }}$ and the characteristic length $\mathcal{L}$.

$$
N u=\frac{\alpha \cdot \mathcal{L}}{\lambda_{\text {air }}}
$$

According to Muzychka and Yovanovich [8], the characteristic length is defined as the square root of the channel's cross section.

Using the analogy between heat and mass transfer, the Sherwood number $S h$ can be calculated using the same Nusselt correlation by substituting the Prandtl number $\mathrm{Pr}$ 
through the Schmidt number $S c$ (eq. (4) and (5)) [3]. The convective mass transfer coefficient $\beta$ is derived from the Sherwood number considering the diffusion coefficient $D_{\mathrm{H}_{2} \mathrm{O}-\text { air }}$ of water vapour in air and the air's density $\rho_{\text {air }}$ (eq. (6)).

$$
\begin{gathered}
S c=P r \\
S h=N u \\
S h=\frac{\beta \cdot \mathcal{L}}{\rho_{\text {air }} \cdot D_{\mathrm{H}_{2} O-\text { air }}}
\end{gathered}
$$

The diffusion coefficient of water vapour in air is calculated using the Chapman-Enskog kinetic theory [9]. With the convective heat and mass transfer coefficients, the heat and moisture transfer in the air ducts is expressed as in eq. (7) and (8). The driving potential for the heat transfer is the difference between the air temperature $T_{i, f / p}$ in the flow and the air temperature $T_{i, f / p, m}$ at the membrane surface. Similarly, the difference between the mass fraction of water vapour $X_{i, f / p}$ in the flow and the mass fraction $X_{i, f / p, m}$ at the membrane surface is the driving potential for moisture transport.

$$
\begin{gathered}
\dot{Q}_{i}=\alpha_{i} \cdot A_{M e m, i} \cdot\left(T_{i, f / p}-T_{i, f / p, m}\right) \\
\dot{m}_{\text {Steam }, i}=\beta_{i} \cdot A_{M e m, i} \cdot\left(X_{i, f / p}-X_{i, f / p, m}\right)
\end{gathered}
$$

\subsection{Modelling approach for membrane's permeance}

As pointed out before the membrane's permeance is declared as an input of the model. Hence, it can be defined without changing the model itself.

As a first approach, we chose a linear dependency on the moisture difference between the two incoming airflows, because the experimental data showed this functional correlation, as it is shown in Fig. 3.

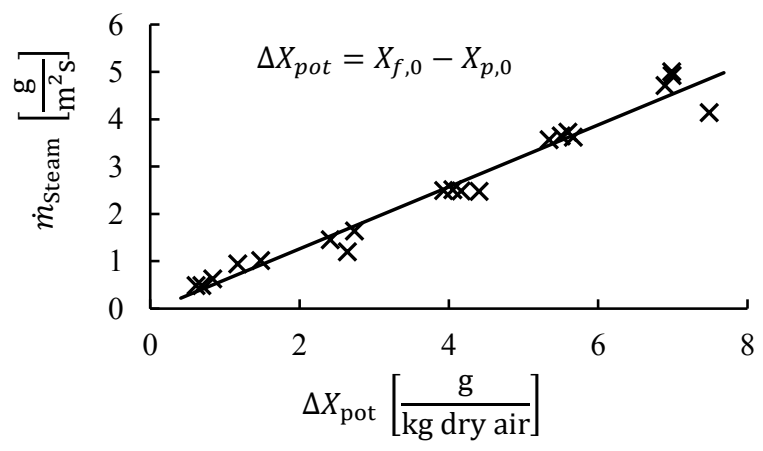

Fig. 3. Mass flow rate through membrane as a function of moisture difference between two incoming airflows

For this reason, the permeance is defined according to eq. (9), where $A$ and $B$ are constant and unknown parameters, which will be fitted afterwards to experimental data, and $\Delta X_{f-p}$ is the moisture difference between the two incoming flows.

$$
P_{i}=A \cdot \Delta X_{f-p}+B
$$

\subsection{Modelling the cross-flow portion}

Enthalpy exchangers in pure counter-current flow arrangement are not common for application. For this reason, it is necessary to apply a cross-flow portion to the model. Koester [3] has modelled the cross-flow portion using a two-dimensional discretization. As the surface distribution of temperature and moisture art not required in our model, we chose a simplified approach based on the Effectiveness-NTU Method [10].

According to [10] the effectiveness of a countercurrent heat exchanger $\varepsilon_{\text {counterflow }}$ can be expressed as shown in eq. (10) and for a cross-flow heat exchanger $\varepsilon_{\text {crosslfow }}$ as shown in eq. (11).

$$
\begin{gathered}
\varepsilon_{\text {counterflow }}=\frac{\mathrm{NTU}}{1+\mathrm{NTU}} \\
\varepsilon_{\text {counterflow }}=\frac{1-\exp \left[-\mathrm{NTU} \cdot\left(1-C_{r}\right)\right]}{1-C_{r} \cdot \exp \left[-\mathrm{NTU} \cdot\left(1-C_{r}\right)\right]} \\
,\left(C_{r}<1\right) \\
\varepsilon_{\text {crossflow }}=1-\exp \left[\frac{\exp \left(-\mathrm{NTU}^{0.78} \cdot C_{r}\right)-1}{\mathrm{NTU}^{-0.22} \cdot C_{r}}\right]
\end{gathered}
$$

A coefficient has been defined (see eq. (12)) by which the calculated transferred heat and moisture is multiplied. In this way, the transferred heat and moisture are reduced for a cross-flow arrangement, because the efficiency correlation for the cross-flow heat exchanger leads always to lower values than the correlation for the counter-flow heat exchanger. The quotient of the two effectiveness correlations leads to a function depending on NTU and the heat capacity flow ratio $C_{r}$. As the volume flow rates for each air side are equal for the most moisture recovery system applications, $C_{r}$ was set to 1.0 . The maximum error by this assumption amounts $0.52 \%$ for a heat capacity flow ratio greater than 0.9 .

$$
C_{\text {cross-flow portion }}=\frac{\varepsilon_{\text {crossflow }}}{\varepsilon_{\text {counterflow }}}
$$

To calculate the heat and moisture transfer for a crosscounter-current enthalpy exchanger the membrane surface is divided into a counter-flow portion and a crossflow portion. Adding the heat and moisture transferred in each surface portion leads to the total heat and moisture transferred.

\section{Results}

In this paragraph, the experimental and simulation results are shown. In the following, we declare the extract air as feed stream and the outdoor air as permeate stream. 


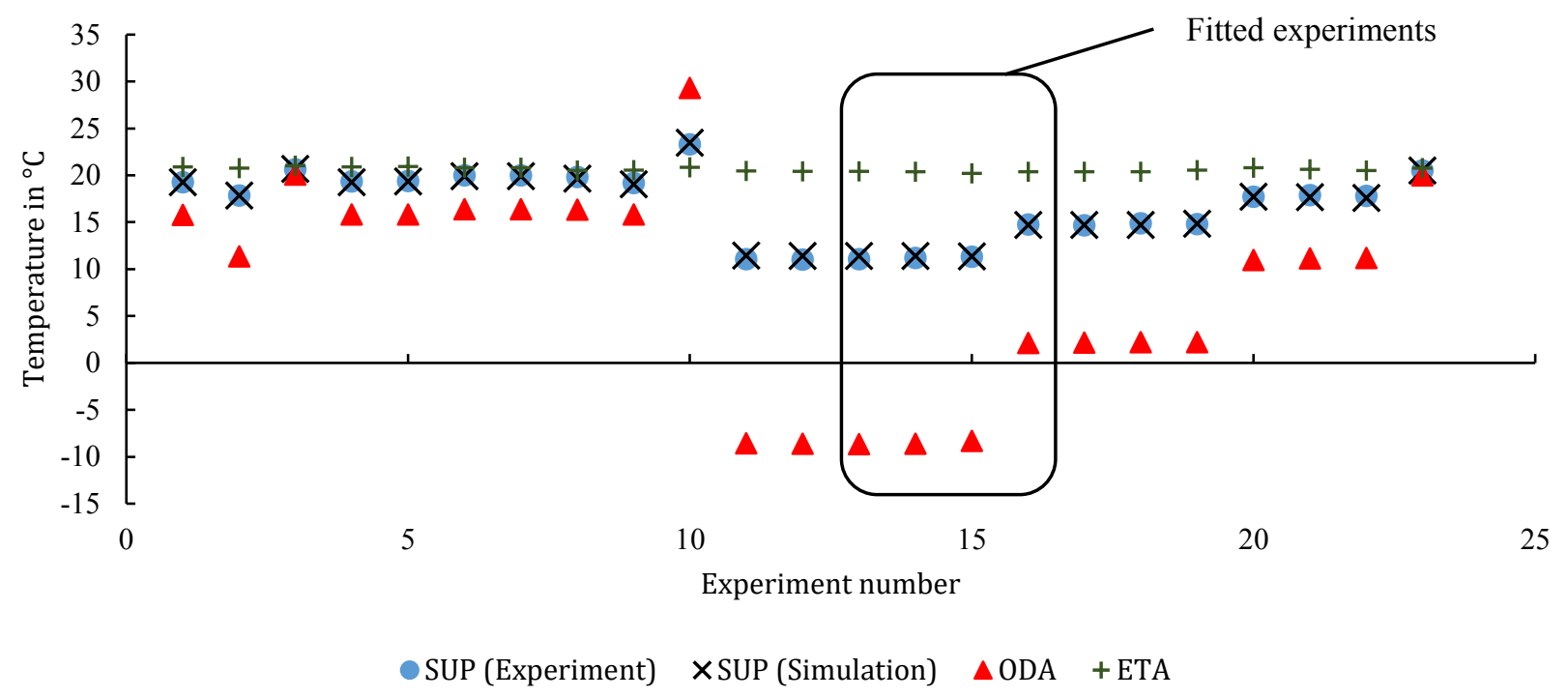

Fig. 4. Measured and simulated supply air (SUP) temperature for different outdoor (ODA) and extract (ETA) air conditions

\subsection{Parameter Fitting}

In Table 1 the fitted parameters and their final value are listed. We fitted the model to the experimental data of four different tests. The tests' boundary conditions are presented in Table 2. Either the temperature or the moisture difference was kept constant.

Table 1. Fitted Parameters.

\begin{tabular}{|r|c|}
\hline $\begin{array}{r}\text { membrane's thermal } \\
\text { conductivity }\end{array}$ & $0.68 \frac{\mathrm{W}}{\mathrm{m} \mathrm{K}}$ \\
\hline parameter $\boldsymbol{A}$ of permeability & $4.49 \cdot 10^{10}$ Barrer $^{1}$ \\
\hline parameter $\boldsymbol{B}$ of permeability & $4.43 \cdot 10^{7}$ Barrer \\
\hline
\end{tabular}

Table 2. Boundary conditions from experimental data

\begin{tabular}{|r|c|c|c|c|}
\hline $\begin{array}{r}\text { test } \\
\text { number }\end{array}$ & 13 & 14 & 15 & 16 \\
\hline $\begin{array}{r}\text { volume } \\
\text { flow rate }\end{array}$ & $400 \frac{\mathrm{m}^{3}}{\mathrm{~h}}$ & $400 \frac{\mathrm{m}^{3}}{\mathrm{~h}}$ & $400 \frac{\mathrm{m}^{3}}{\mathrm{~h}}$ & $400 \frac{\mathrm{m}^{3}}{\mathrm{~h}}$ \\
\hline$\Delta \boldsymbol{T}_{\boldsymbol{f}-\boldsymbol{p}}$ & $29 \mathrm{~K}$ & $29 \mathrm{~K}$ & $29 \mathrm{~K}$ & $18 \mathrm{~K}$ \\
\hline$\Delta \boldsymbol{X}_{\boldsymbol{f}-\boldsymbol{p}}$ & $4,2 \frac{\mathrm{g}}{\mathrm{kg}}$ & $5,6 \frac{\mathrm{g}}{\mathrm{kg}}$ & $7 \frac{\mathrm{g}}{\mathrm{kg}}$ & $7 \frac{\mathrm{g}}{\mathrm{kg}}$ \\
\hline
\end{tabular}

The parameter fitting was carried out with an evolutionary algorithm minimizing the root mean square error (RMSE).

\subsection{Comparison of experimental data with simulation results}

Using the parameter shown in Table 1, the model was simulated with the boundary conditions of in total 23 experimental investigations including the four tests used for the fitting. In the experimental investigations, we set the volume flow rate to 400 and $200 \mathrm{~m}^{3} / \mathrm{h}$ and varied the temperature and humidity difference between the two incoming airflows.

Fig. 4 shows the measured and simulated temperatures at the two inlets and supply air's outlet of the enthalpy exchanger. There is good agreement between the experimentally measured and simulated supply air temperature. This is underlined by the RMSE listed in Table 3.

In Fig. 5, the measured and simulated supply air humidity is shown for the different boundary conditions. Again, the simulation results fit the measurements quite well in general. Yet for experiment number 9 the simulation does not match the measurement data. The moisture recovered in this experiment was rather low for the high driving potential between outdoor and extract air. Comparing experiment 9 with other experiments under similar conditions, the measured value of the supply air humidity is lower. Therefore, we assume that errors such as an inhomogeneous inflow occurred during experiment 9. This leads to a low reliance of this measured humidity. There is also a higher divergence between simulation and experiment, when the humidity difference between outdoor air and extract air is low. This is caused by the measurement error of the humidity sensors $(+/-3.5 \% \mathrm{rH})$. Consequently, the low driving potential is not well determined.

${ }^{1} 1$ Barrer $=3.35 \cdot 10^{-16} \frac{\mathrm{mol} \cdot \mathrm{m}}{\mathrm{m}^{2} \cdot \mathrm{s} \cdot \mathrm{Pa}}[11]$ 


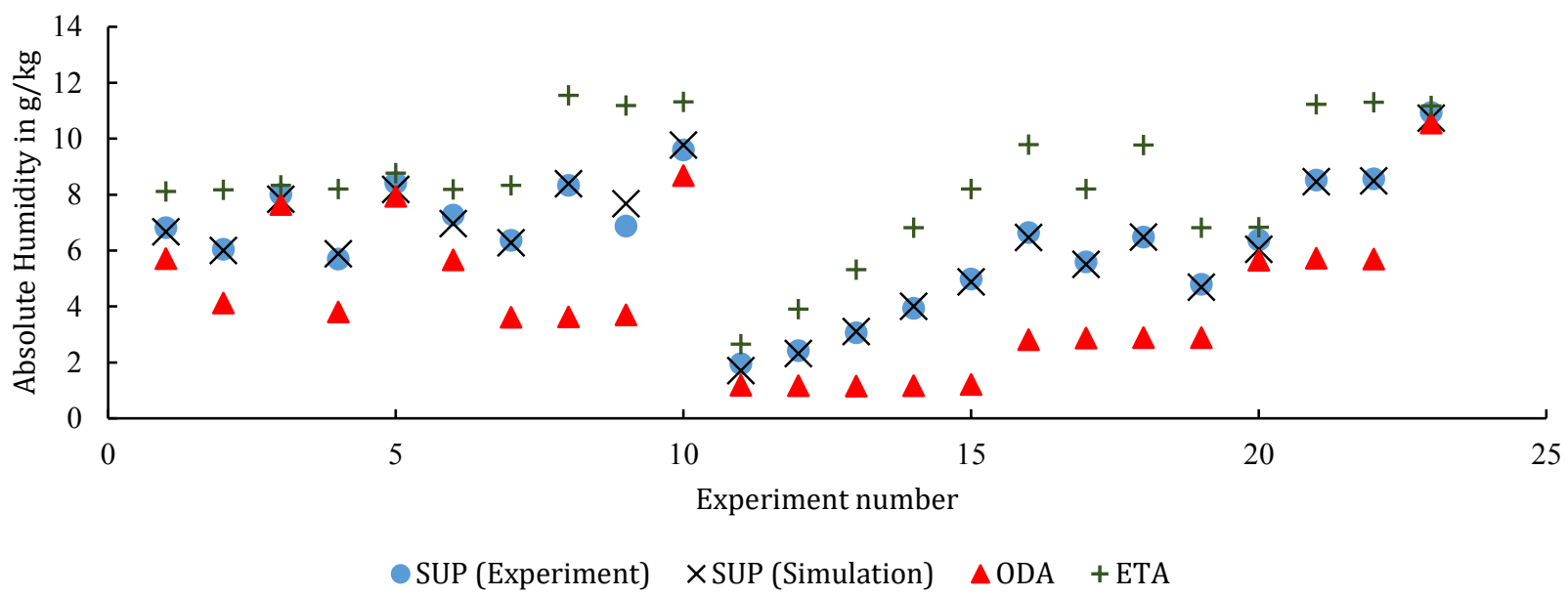

Fig. 5. Measured and simulated supply air humidity for different outdoor (ODA) and extract (ETA) air conditions

Table 3. Root mean square error for temperature and humidity

\begin{tabular}{|l|c|c|}
\hline \multirow{2}{*}{} & \multicolumn{2}{|c|}{ RMSE } \\
\cline { 2 - 3 } & fitting & total \\
\hline $\begin{array}{l}\text { supply air } \\
\text { temperature }\end{array}$ & $0.18 \mathrm{~K}$ & $0.16 \mathrm{~K}$ \\
\hline $\begin{array}{l}\text { supply air } \\
\text { humidity }\end{array}$ & $0.11 \mathrm{~g} / \mathrm{kg}$ & $0.23 \mathrm{~g} / \mathrm{kg}$ \\
\hline
\end{tabular}

The RMSE (see Table 3) of the supply air humidity underlines a good accordance of the simulation. The RMSE is lower than the mean measurement error of the absolute humidity due to error propagation $(+/-0.4 \mathrm{~g} / \mathrm{kg})$.

The operation of an enthalpy exchanger is described by two characteristic values, the sensible and latent efficiency defined as shown in equation (13) and (14). In the following, these values are compared for the experimental data and simulation results.

$$
\begin{gathered}
\varepsilon_{\text {sensible }}=\frac{T_{\mathrm{SUP}}-T_{\mathrm{ODA}}}{T_{\mathrm{ETA}}-T_{\mathrm{ODA}}} \\
\varepsilon_{\text {latent }}=\frac{X_{\mathrm{SUP}}-X_{\mathrm{ODA}}}{X_{\mathrm{ETA}}-X_{\mathrm{ODA}}}
\end{gathered}
$$

The sensible efficiency is shown in Fig. 6. The simulation model leads to similar sensible efficiencies for similar driving temperature potentials. The greatest deviation is observed for low temperature potentials. Since the temperature potential is in the denominator of the sensible efficiency's definition, the error of the calculation from the measurement data is rather high (up to 0.09 ).

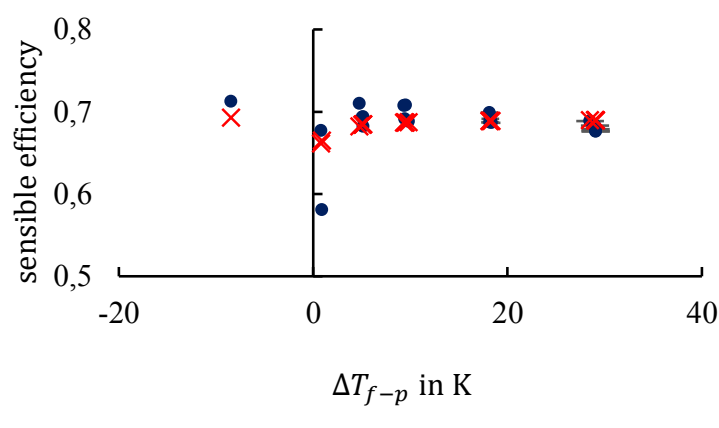

- Experiment $\times$ Simulation

Fig. 6. Measured and simulated sensible efficiency depending on temperature difference $\Delta T_{f-p}$ between extract and outdoor air

Fig. 7 shows the calculated enthalpy exchanger's latent efficiency (see eq. (14)) from the simulation results and measurement data. The error due to error propagation in determining the latent efficiency from the measurement data is also shown. The error is highest for low moisture differences between the outdoor and extract air. Except for one value, the simulation results lie within the error limits.

Table 4. RMSE for sensible and latent efficiency

\begin{tabular}{|l|c|c|}
\hline \multirow{2}{*}{} & \multicolumn{2}{|c|}{ RMSE } \\
\cline { 2 - 3 } & fitting & total \\
\hline $\begin{array}{l}\text { sensible } \\
\text { efficiency }\end{array}$ & 0.0725 & 0.0238 \\
\hline $\begin{array}{l}\text { latent } \\
\text { efficiency }\end{array}$ & 0.1265 & 0.132 \\
\hline
\end{tabular}




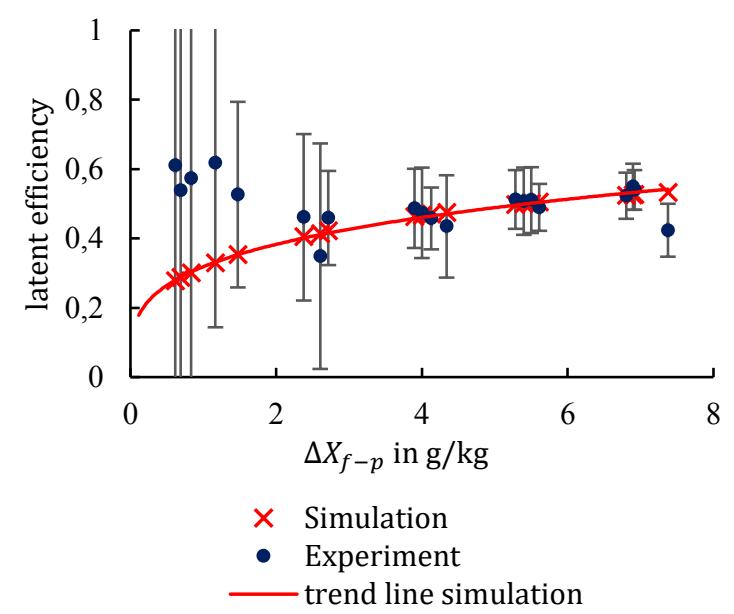

Fig. 7. Measured and simulated latent efficiency depending on moisture difference $\Delta X_{f-p}$ between extract and outdoor air

The quality of the simulation results is also represented by the RMSE (see Table 4). The deviation of the simulation results from the measured data is small for the sensible efficiency. The latent efficiency is not predicted well, as the RMSE is rather high. This is caused by the discrepancy between simulation and experiment for low moisture potentials. For these values, a new validation with measurements at higher measuring accuracy will be necessary.

\section{Conclusion and outlook}

The comparison between the measured temperatures and humidities and the simulation results has shown that the model is able to predict the enthalpy exchanger's operation for different boundary conditions and volume flow rates adequately with an RMSE of $0.16 \mathrm{~K}$ for the temperature and $0.23 \mathrm{~g} / \mathrm{kg}$ for the humidity.

Moreover, the enthalpy exchanger's sensible efficiency is mapped well by the simulation within the limits of the measurement accuracy. Higher deviations, especially for low moisture differences, occur in the latent efficiency.

Due to the low accuracy of the humidity sensors, the model could not be validate so far for low moisture differences between the two incoming airflows. For this reason, the test rig will be equipped with new humidity sensors, which have a higher accuracy. The experiments with low moisture differences will be repeated.

To perform a validation of the model's dynamics, experiments will be carried out testing load change of the volume flow rate, start-up and shutdown processes.

Grateful acknowledgement is made for financial support by the Federal Ministry for Economic Affairs and Energy (BMWi) and the Federation of Industrial Research Associations (AIF), promotional reference $25 \mathrm{EWN} / 1$.

\section{References}

1. L.-Z. Zhang, C.-H. Liang, L.-X. Pei. J. Membr. Sci. 325, 672-682 (2008)
2. S. Dugaria, L. Moro, D. Del Col. J. Phys.: Conf. Ser. 655 (2015)

3. S. Koester. Membrane-based Enthalpy Exchangers. (2016)

4. Dassault Systemes. Dymola Multi-Engineering Modeling and Simulation, https://www.3ds.com/productsservices/catia/products/dymola

5. Modelica Association. https://www.modelica.org/

6. D. Müller, M. Lauster, A. Constantin, M. Fuchs, P. Remmen. AixLib - An Open-Source Modelica Library within the IEA-EBC Annex 60 Framework. BauSIM 2016,.3-9 (2016)

7. J. G. Wijmans, R. W. Baker. J. Membr. Sci. 107, 1$21(1995)$

8. Y. S. Muzychka, M. M. Yovanovich. J. Heat. Transf. 126, 54-61 (2004)

9. J. H. Lienhard, J. H. Lienhard. A Heat Transfer Textbook. (2008)

10. F. P. Incropera, D. P. DeWitt, T. L. Bergman, A. S. Lavine. Fundamentals of Heat and Mass Transfer. (2007)

11. P. Kjeldsen. Wat. Res. 27, 121-131 (1993) 